\title{
Quantitative speciation of sulfur in bacterial sulfur globules: X-ray absorption spectroscopy reveals at least three different species of sulfur
}

\author{
Alexander Prange, ${ }^{1}$ Reinhold Chauvistré, ${ }^{2}$ Hartwig Modrow, ${ }^{2}$ \\ Josef Hormes, ${ }^{2}+$ Hans G. Trüper ${ }^{1}$ and Christiane Dahl ${ }^{1}$
}

Author for correspondence: Christiane Dahlł. Tel: +49 228 732119. Fax: +49228737576. e-mail:ChDahl@uni-bonn.de

\footnotetext{
1 Institut für Mikrobiologie \& Biotechnologie, Rheinische FriedrichWilhelms-Universität Bonn, Meckenheimer Allee 168, D-53115 Bonn, Germany

2 Physikalisches Institut, Rheinische FriedrichWilhelms-Universität Bonn Nußallee 12, D-53115 Bonn, Germany
}

\begin{abstract}
X-ray absorption near edge structure (XANES) spectroscopy at the sulfur Kedge was applied to probe the speciation of sulfur of metabolically different sulfur-accumulating bacteria in situ. Fitting the spectra using a least-square fitting routine XANES reveals at least three different forms of sulfur in bacterial sulfur globules. Cyclooctasulfur dominates in the sulfur globules of Beggiatoa alba and the very recently described giant bacterium Thiomargarita namibiensis. A second type of sulfur globules is present in Acidithiobacillus ferrooxidans: here the sulfur occurs as polythionates. In contrast, in purple and green sulfur bacteria the sulfur mainly consists of sulfur chains, irrespective of whether it is accumulated in globules inside or outside the cells. These results indicate that the speciation of sulfur in the sulfur globules reflects the different ecological and physiological properties of different metabolic groups of bacteria.
\end{abstract}

Keywords: XANES, sulfur K-edge, quantitative analysis, sulfur-oxidizing bacteria, sulfur globules

\section{INTRODUCTION}

Reduced inorganic sulfur compounds like sulfide or thiosulfate are oxidized by different 'sulfur bacteria'. In most cases the sulfur compounds are oxidized to sulfate and used as electron donors for anoxygenic photosynthesis or for aerobic and anaerobic chemotrophic growth. Furthermore, several bacteria are able to detoxify sulfide by oxidation. In many cases sulfur globules of 'elemental sulfur' are formed as an intermediate of reduced sulfur compound oxidation and deposited either inside (e.g. Chromatiaceae, Beggiatoa spp.) or outside (e.g. Ectothiorhodospiraceae, Chlorobiaceae) the cells. Since the early work of C. Cramer, F. Cohn and S. Winogradsky in the 19th century (Cramer, 1870; Cohn, 1875; Winogradsky, 1887), numerous different techniques have been used to determine the

\footnotetext{
† Present address: The John Bennett Johnston, Sr Center for Advanced Microstructures \& Devices (CAMD), Louisiana State University, 6980 Jefferson Highway, Baton Rouge, LA 70806, USA.

$\ddagger$ Correspondence on the physical aspects of the work should be sent to Hartwig Modrow (Tel: +49 228 733203. Fax: +49 228737869 . e-mail: Modrow@physik.uni-bonn.de).
}

Abbreviation: XANES, X-ray absorption near edge structure. exact chemical nature of the stored sulfur (Trüper \& Hathaway, 1967; Hageage et al., 1970; Schmidt et al., 1971; Guerrero et al., 1984; Steudel, 1989; Steudel et al., 1990; Steudel \& Albertsen, 1999). In all investigations carried out so far, the sulfur was extracted from the bacterial cells before analysis. Extraction with polar or nonpolar organic solvents as well as the potential presence of oxygen during extraction may, however, cause dramatic changes in the structure of sulfur. For instance, if the sulfur is present in an unstable modification, it may be transformed e.g. into cyclooctasulfur, the thermodynamically most stable sulfur species at ambient temperature and pressure (Donohue, 1974; Steudel et al., 1990; Steudel, 1996).

$\mathrm{X}$-ray absorption near edge structure (XANES) spectroscopy at the sulfur K-edge using synchrotron radiation is an excellent tool to study the speciations of sulfur in biological samples (Rompel et al., 1998; Pickering et al., 1998; Prange et al., 1999, 2001). The method is non-destructive and measurements can be performed in situ (e.g. aquatic environmental samples and cultured bacteria in liquid media). XANES allows the determination of the valence of an exited atom as well as the electronegativity of neighbouring atoms (Bianconi, 1988). In addition, the lengths of sulfur chains 
can be determined at least in a given range $(\leqslant 3$ sulfur atoms) (Chauvistré et al., 1997). Furthermore, XANES spectra can yield information on the type of the chemical bond in the second coordination shell of the excited sulfur atom (e.g. C-C single, double or triple bonds). The fact that the local environment of the absorbing atoms is probed implies that XANES spectra are additive, i.e. the spectrum of a mixture of substances $\mathrm{A}$ and $\mathrm{B}$ can be composed from the separately measured spectra of A and B. This additivity is the basis for the 'quantitative analysis' of XANES spectra which means the decomposition of a sum spectrum into the components it is composed of. To achieve this decomposition, a 'quality function' defined by the difference between experimental data and a linear combination of spectra contained in a basis set can be minimized (e.g. Modrow et al., 2001).

Using XANES spectroscopy as a 'fingerprint method', comparing the obtained spectra with those of reference compounds, we recently found evidence for the presence of long sulfur chains ( $>3$ sulfur atoms), most probably terminated by $\mathrm{C}$ or $\mathrm{H}$ atoms, in different phototrophic sulfur bacteria (Prange et al., 1999). In contrast, Pickering et al. (2001) very recently concluded on the basis of theoretical considerations that the form of sulfur that most resembles the globule sulfur in metabolically different sulfur-accumulating bacteria is 'simple, solid $\mathrm{S}_{8}$ '. This conclusion contradicts a number of previous experimental results (Hageage et al., 1970; Guerrero et al., 1984; Mas \& van Gemerden, 1987). In the experimental study presented here, we reach a different conclusion which is in good agreement with previous experimental results. The present study aimed at clarifying whether different sulfur species are present in physiologically and phylogenetically diverse sulfuroxidizing bacteria. A least-square fitting routine was used to determine possible contributions of different reference compounds to the bacterial spectra, thereby allowing a quantitative analysis of sulfur in the bacterial sulfur globules.

\section{METHODS}

Bacterial strains, media, growth conditions. Allochromatium vinosum DSMZ $180^{\mathrm{T}}$ [formerly Chromatium vinosum (Imhoff et al., 1998)], Thiocapsa roseopersicina DSMZ 219, Marichromatium purpuratum [formerly Chromatium purpuratum (Imhoff et al., 1998)] DSMZ $1591^{\mathrm{T}}$ and Chlorobium vibrioforme (f. thiosulfatophilum) DSMZ 263 were cultured photolithoautotrophically on sulfide (Pfennig \& Trüper, 1992). Halorhodospira halophila DSMZ $244^{\mathrm{T}}$ and Halorhodospira abdelmalekii DSMZ 2110 were grown photoautotrophically on sulfide (Then \& Trüper, 1983; Imhoff, 1992). Sulfide solution for feeding (1-4 mM) was prepared according to the procedure of Siefert \& Pfennig (1987). Acidithiobacillus ferrooxidans DSMZ 584 [formerly Thiobacillus ferrooxidans (Kelly \& Wood, 2000)] was grown on thiosulfate (DSMZ medium no. 71). Beggiatoa alba DSMZ 1416 was cultivated on Beggiatoa medium (DSMZ medium no. 155, but without catalase) and fed with $1 \mathrm{mM}$ sulfide solution (see above). Cells were prepared for measurement as described previously (Prange et al., 1999). Thiomargarita namibiensis, collected from Namibian shelf sediments (Schulz et al., 1999), was kindly donated by Dr Heide Schulz (Max-Planck-Institute for Marine Microbiology, Bremen).

Preparation of sulfur globules. Sulfur globules of Allochromatium vinosum grown photoautotrophically on sulfide were prepared according to the procedure of Schmidt et al. (1971) and Brune (1995). The cells were disintegrated by ultrasonification, the sulfur globules were sedimented in a centrifuge and the supernatant was removed with a pipette.

\section{X-ray absorption spectroscopy}

Experimental. XANES spectra were recorded at beamline BN3 using synchrotron radiation from the electron stretcher accelerator (ELSA) of the Institute of Physics at the University of Bonn (Althoff et al., 1990). The storage ring was operated at an energy of $2 \cdot 3 \mathrm{GeV}$ with electron currents between 20 and $100 \mathrm{~mA}$. The synchrotron radiation was monochromatized by a modified Lemonnier-type double-crystal X-ray monochromator (Lemonnier et al., 1978) equipped with InSb (111) crystals. The monochromatic flux rate per second on the sample was about $10^{9}$ photons (at $50 \mathrm{~mA}$ ). Measurements were performed in transmission mode with ionization chambers (60 mbar air inside), measuring the beam intensities in front of and behind the sample. For energy calibration of the spectra, the spectrum of $\mathrm{ZnSO}_{4}$ was used as a 'secondary standard' setting the maximum of the first resonance (white line) to an energy of $2481 \cdot 4 \mathrm{eV}$. According to the step width, this value is reproducible to $\pm 0 \cdot 1 \mathrm{eV}$. Further details of the experimental procedure have been published previously (Winter et al., 1995; Chauvistré et al., 1997). Spectra were scanned with step widths of $0.6 \mathrm{eV}$ in the pre-edge region between 2450 and $2460 \mathrm{eV}, 0.09 \mathrm{eV}$ between 2460 and $2490 \mathrm{eV}$, the main region of interest, and $0.2 \mathrm{eV}$ between 2490 and $2510 \mathrm{eV}$. Typical integration times were $500-1000$ ms per point. A linear background determined in the pre-edge region was subtracted from the raw data to correct the absorption from higher shells and from supporting materials. Spectra were normalized at $2490 \mathrm{eV}$, where the variation of the absorption cross-section is already very small. In some cases, spectra were normalized to the maximum of the white line for a better comparison e.g. of energy positions.

MINUIT fitting of spectra. The interactive fitting and plotting package Mn-Fit 4.04/15 was used (available at: http://wwwzeus.physik.uni-bonn.de/ brock/mn_fit.html). Mn-Fit 4.04/15 uses the function minimization tool 'MINUIT' which is part of the CERNlib available at CERN (http:// wwwinfo.cern.ch/asdoc/minuit/node2.html) to fit histograms or data. MINUIT is a tool to find the minimum value of a multi-parameter function, and it analyses the shape of the function around the minimum. In our case, a chi-square function was used to compute the best fit parameter values. For a set of reference spectra, MINUIT finds a linear combination of these spectra which reproduces the XANES spectra with the highest probability. Non-statistical errors may occur from an incomplete set of reference spectra or from reference spectra not monitoring exactly the local sulfur environment. The error of the percentage contributions of sulfur (Tables 1, 2 and 3) can be estimated to be $\pm 10 \%$.

Reference compounds. Cysteine, cystine, reduced glutathione, oxidized glutathione, sodium thiosulfate, sodium tetrathionate, cyclooctasulfur $\left(\mathrm{S}_{8}\right.$ rings $)$, zinc sulfate and polymeric sulfur (kindly provided by Professor Ralf Steudel, Technical University of Berlin), were used as reference compounds for fitting the spectra. These compounds were of reagent grade, purchased (unless otherwise stated) from Sigma and used as 
received. Mixtures of reference compounds, which were used in order to validate the stability of the results obtained by the fitting approach discussed above, were prepared by careful simultaneous grinding of weighed shares of the ingredients using a pestle and mortar. The solid reference compounds were ground into fine powder and put homogeneously on a self-adhesive kapton film (DuPont). The thickness of the samples was optimized in order to avoid both possible thickness- and pinhole-effects

\section{RESULTS AND DISCUSSION}

Following our earlier investigations on purple sulfur bacteria using XANES fingerprints (Prange et al., 1999), it was one goal of this study to probe the speciations of sulfur in metabolically and phylogenetically different phototrophic and chemotrophic sulfur bacteria. A second goal was to quantitatively analyse the spectra (see below). For these analyses, a broad variety of reference compounds for sulfur in different speciations (Fig. 1) and defined mixtures of reference compounds (Fig. 2) were measured and their respective relevance tested. In general, it should be noted that these compounds are used only as representatives for a given class of an atomic environment of the sulfur atom. For example, zinc sulfate crystals were used as a reference compound (Fig. 1). While it is obvious that these crystals are not present in the bacteria we studied, this compound was used as a proxy for sulfate anions. It thus allows tracing of the presence of sulfate in general. In principle, it is possible to achieve a high degree of speciation using XANES. This can be recognized by the fact that in some cases a small energy shift is observed between fit and experimental data in the energy region which is characteristic for a sulfate (see Fig. 3). Another example for this fact can be extracted from Fig. 1, where clear differences between the spectra of the reference compounds for a $\mathrm{C}-\mathrm{S}-\mathrm{H}$ environment are observed. However, this same observation has different implications in the two cases, because in the sulfate case only sulfates can contribute to structures in this energy region, whereas the $\mathrm{C}-\mathrm{S}-\mathrm{H}$ environment can interfere strongly with $\mathrm{C}-\mathrm{S}-\mathrm{C}$ and even $\mathrm{S}-\mathrm{S}$ contributions. Thus it is crucial to include a variety of corresponding references in the basis set or at least to run tests including such reference compounds. It turns out that in most cases a clear rejection of all representatives but one of a given environment occurs (see examples below). Conversely, the removal of the 'correct' compound from the basis leads to a dramatic deterioration of the fit. In order to prove the presence of a specified compound in a bacterium, it would be necessary that XANES spectra are unique in a strict sense. However, it is possible to limit the number of candidates sufficiently to allow the development of a hypothetical sulfur metabolism if this can be supported by biological considerations and does not contradict other experimental results. S K-edge XANES spectra of compounds which seem to be representative for the system 'bacterial sulfur globule' and yield the best fits (Fig. 1) were used for fitting the S K-edge XANES spectra of the bacteria to specify the forms of sulfur in a quantitative manner.

\section{Quantitative analysis of XANES spectra}

As mentioned above, XANES measurements provide a sensitive fingerprint of the local environment of an absorbing atom in situ. It is often neglected that the sensitivity of XANES is an ambiguous feature. On the one hand, it allows different sulfur-containing substances to be distinguished clearly from each other. However, this implies on the other hand the need for extremely careful selection of reference compounds for the basis set, because e.g. variations of the organic residues $\mathrm{R}$ of a substance with the structure $\mathrm{R}-\mathrm{S}_{n}-\mathrm{R}$ can influence XANES spectra considerably (Chauvistré et al., 1997; H. Modrow \& J. Hormes, unpublished). As a consequence, selection of inadequate basis compounds can lead to results the reliability of which is at least questionable. This problem is remedied by the fact that the bacterial spectra discussed here involve references in which sulfur is present in different formal oxidation states whose most prominent features are clearly separated from each other on the energy scale (Fig. 1). On the other hand, it must be noted that the fit has to cover an energy range of $20 \mathrm{eV}$. Thus, it has to take into account not only the so-called white lines (first strong resonances in the spectra) but also the shape resonances, which are strongly dependent on the geometric environment of the absorber atom including the mid-range order. However, the mid-range geometric environment even of a pure crystalline reference substance can be expected to be different in the real sulfur globule due to the lack of long-range order.

\section{Verification of the quantitative analysis}

In order to verify our approach, we performed analysis of a defined mixture composed of cystine $(47 \%)$, cysteine $(24 \%)$, reduced glutathione $(22 \%)$ and oxidized glutathione $(7 \%)$. Such a mixture presents a demanding example because the white lines of these substances are quite similar in shape and overlap considerably (Fig. 1). The results of the quantitative analysis are given in Table 1 and Fig. 2(a). Evidently, the results are independent of the energy range the fitting is performed on, which indicates that stable background correction and normalization procedures have been employed. This is also immediately clear when looking at Fig. 2(a), as the fits reproduce the shape of the experimental data equally well for each energy range used in the fitting routine. It should be stressed that this is true even if we restrict ourselves exclusively to the 'electronic information' provided by the white line. In Fig. $2(\mathrm{a})$ it is also apparent that experimental data and the fit using the longest energy range run in parallel to each other, which again shows the stability of the background subtraction procedures.

We would like to strengthen further the case for the stability and reliability of our results against the background of the previously mentioned paper dealing with X-ray absorption spectroscopy on sulfur bacteria by Pickering et al. (2001). In this paper, it is erroneously assumed that there is no difference between the $\mathrm{S} \mathrm{K}$-edge 

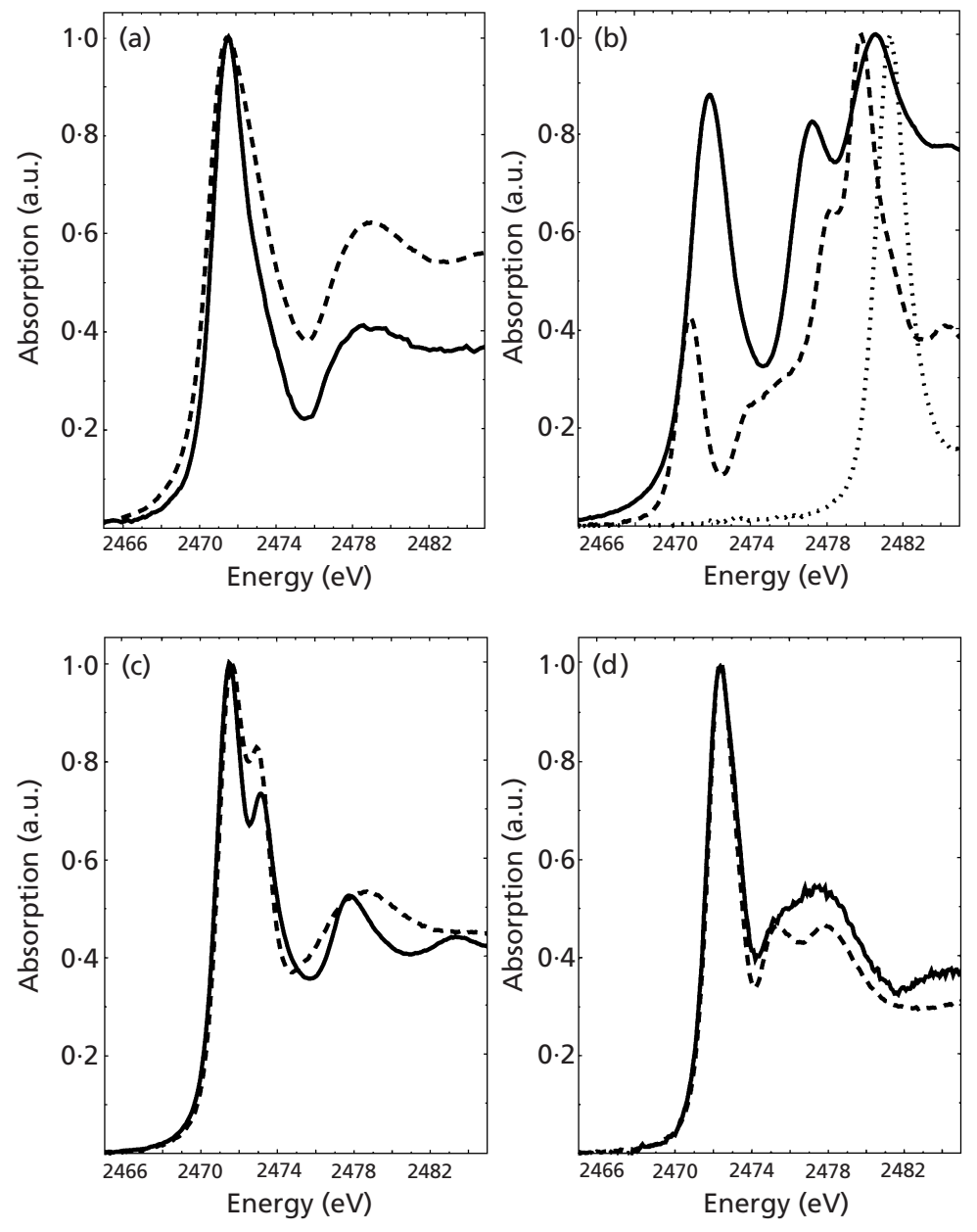

Fig. 1. $\mathrm{S} K$-edge $X A N E S$ spectra of the reference compounds which were used for fitting bacterial spectra: (a) polymeric sulfur (solid line) and cyclooctasulfur ( $S_{8}$ rings) (broken line); (b) sodium tetrathionate (solid line), sodium thiosulfate (broken line) and zinc sulfate (dotted line); (c) cystine (solid line) and oxidized glutathione (broken line); (d) cysteine (solid line) and reduced glutathione (broken line). Spectra were normalized on the maximum of the white line. a.u., arbitrary units.

XANES spectra of carefully ground cyclooctasulfur and polymeric sulfur, but that the observed differences between these spectra are due to experimental artefacts. If this were true, it should not be possible to perform a correct quantitative analysis of a mixture of these two compounds. To test this presumption, we analysed two independently prepared defined mixtures of cyclooctasulfur $(25 \%)$ and polymeric sulfur $(75 \%)$. MINUIT fits of the spectra obtained covering two different energy ranges were performed (Fig. 2b, c, Table 2) yielding correct results with errors of only $\leqslant 5 \%$. Pickering et al. (2001) assume that the reduced white line of cyclooctasulfur is induced by pinhole effects. As we find that the white line of the spectrum of polymeric sulfur is comparatively much more intense (Fig. 1a) one would have to assume that pinhole effects do not significantly influence the spectrum in this case. Based on this hypothesis, pinhole effects should be reduced in a mixture of cyclooctasulfur and polymeric sulfur. Consequently, the intensity of the white line of such a mixture would have to be increased, thus resulting in artificially high amounts of polymeric sulfur after quantitative analysis. To accommodate this increase, the contribution of polymeric sulfur would have to be overestimated by the fitting routine. However, we do not find evidence for such an effect (Table 2). From Fig. 2(b, c) it is evident that the fitting of the white line alone is again sufficient to extract the relative contributions of the different references. This is especially important as possible thickness or pinhole effects affect resonances of different intensity to a different extent (Parrat et al., 1957; Stern \& Kim, 1981). Consequently, the stability of the fitting results with respect to the energy range of the fit supports the conclusion that we are not dealing with experimental artefacts. Again, including an increased energy range in the fitting routine does not affect the fit significantly and the long-range fit runs in parallel to the experimental data in both cases, proving yet again the stability of the background treatment. Fig. 2(d) shows the residuum between the experimental spectra (spectrum sample 2 minus spectrum sample 1 ) displayed in Fig. 2(b, c), suggesting an explanation for the slightly different results. It is evident from this figure that, both in the area of the white line and the shape resonances, the difference between the spectra exceeds the noise level significantly. We attribute this fact, which we also encountered with other reference mixtures, to slight inhomogeneities of the distribution of the various compounds in the sample which remain even after a careful mixing procedure. 

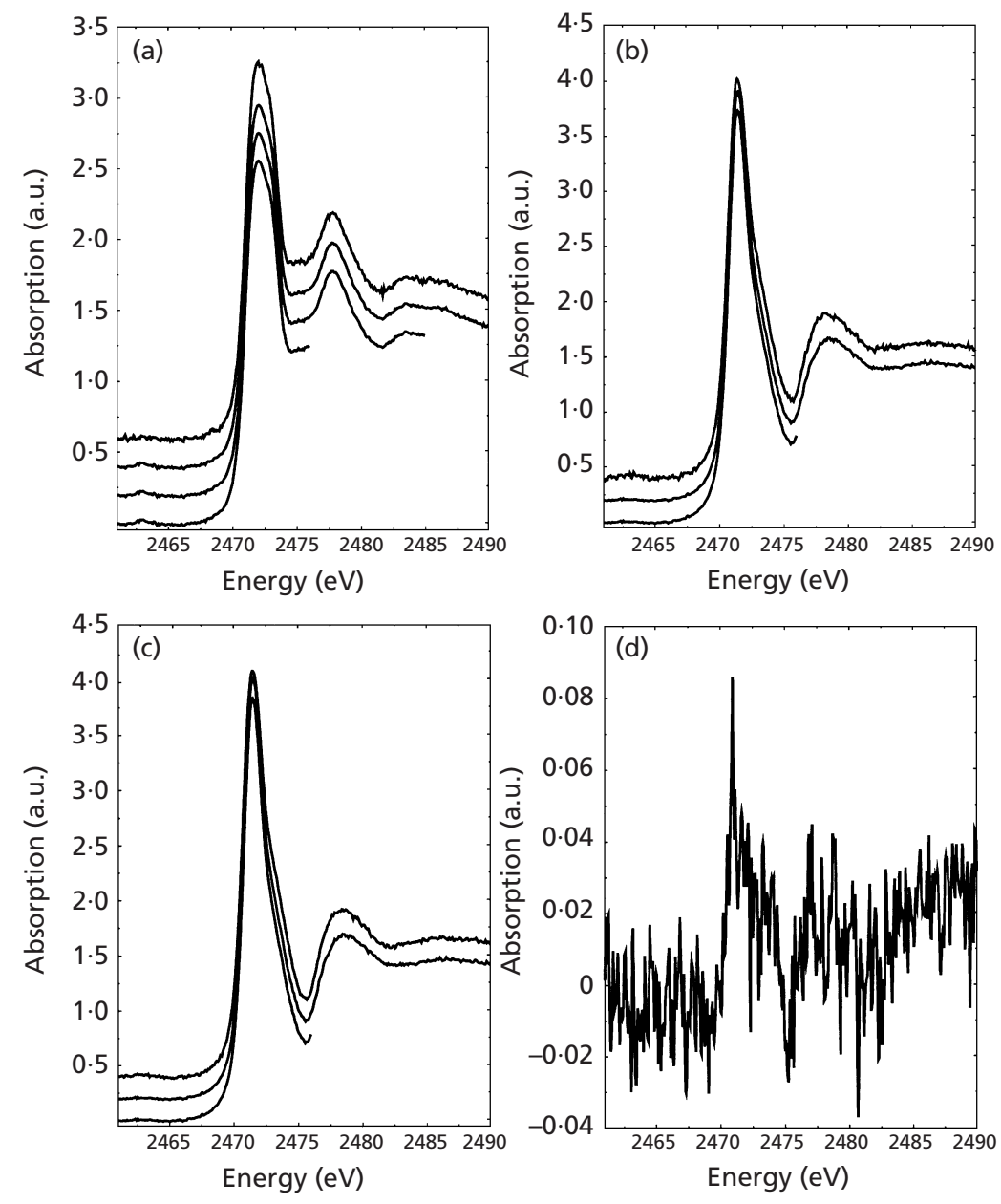

Fig. 2. $(a-c)$ The top trace in each panel shows, respectively, the $S$ K-edge XANES spectrum of: (a) a mixture of cystine $(47 \%)$, cysteine $(24 \%)$, reduced glutathione ( $22 \%)$ and oxidized glutathione (7\%); (b) sample 1 of the mixture of cyclooctasulfur $(25 \%)$ and polymeric sulfur $(75 \%)$; (c) sample 2 of the mixture of cyclooctasulfur $(25 \%)$ and polymeric sulfur $(75 \%)$. The lower traces in each panel show MINUIT fits corresponding to the accompanying $\mathrm{S}$ K-edge XANES spectrum of different energy ranges (from top to bottom): (a) 2461-2490 eV, 2461$2485 \mathrm{eV}, 2461-2476 \mathrm{eV}$; (b, c) 2461-2490 eV, 2461-2476 eV. Panel (d) shows a difference spectrum (spectrum sample 2 minus spectrum sample 1) derived from the two $S$ K-edge XANES spectra (top traces in $b$ and c). a.u., arbitrary units.

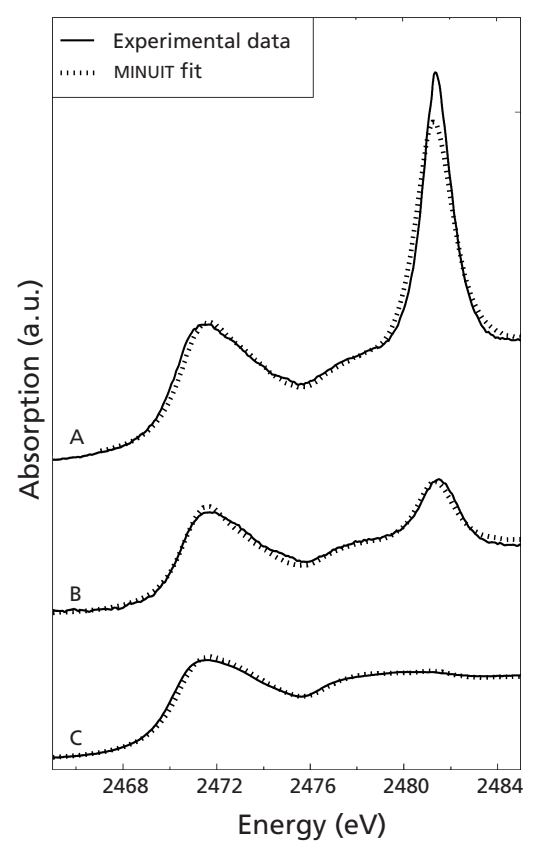

Fig. 3. $\mathrm{S}$ K-edge XANES spectra of Thiomargarita namibiensis cells without salt water (A), $T$. namibiensis cells in natural salt water (b) and Beggiatoa alba (c), and accompanying MINUIT fits. a.u., arbitrary units.
Table 1. Results of fitting the $S K$-edge XANES spectrum of a defined mixture of cystine, cysteine, reduced glutathione and oxidized glutathione using different energy ranges

See also Fig. 2(a).

\begin{tabular}{|lcccc|}
\hline & \multicolumn{4}{c|}{ Percentage composition } \\
\cline { 2 - 5 } & Cystine & Cysteine & GSH* $^{*}$ & GSSG* \\
\hline Actual composition & 47 & 24 & 22 & 7 \\
Results of fitting & & & & \\
$2461-2476 \mathrm{eV}$ & 47 & 27 & 17 & 8 \\
$2461-2481 \mathrm{eV}$ & 48 & 27 & 18 & 7 \\
$2461-2490 \mathrm{eV}$ & 48 & 26 & 18 & 7 \\
\hline
\end{tabular}

*GSH, reduced glutathione; GSSG, oxidized glutathione.

\section{Sulfur as sulfur rings (cyclooctasulfur)}

In Fig. 3, the S K-edge XANES spectra of Thiomargarita namibiensis are shown together with their accompanying MINUIT fits. Both species appear to thrive best under microoxic or anoxic conditions, although atmospheric oxygen conditions are not toxic (Schulz et 
Table 2. Results of fitting the $S$ K-edge XANES spectra of two samples of a defined mixture composed of cyclooctasulfur and polymeric sulfur on two energy ranges

See also Fig. 2(b, c).

\begin{tabular}{|lcc|}
\hline & \multicolumn{2}{c|}{ Percentage composition } \\
\cline { 2 - 3 } & $\begin{array}{c}\text { Polymeric } \\
\text { sulfur }\end{array}$ & Cyclooctasulfur \\
\hline Actual composition & 75 & 25 \\
Results of fitting & 70 & 30 \\
Sample 1, 2461-2476 eV (Fig. 2b) & 71 & 29 \\
Sample 2, 2461-2476 eV (Fig. 2c) & 72 & 28 \\
Sample 1, 2461-2490 eV (Fig. 2b) & 72 & 28 \\
Sample 2, 2461-2490 eV (Fig. 2c) & & \\
\hline
\end{tabular}

Table 3. Results of fitting the S K-edge XANES spectra of different bacteria to the sum of different reference spectra

See also Figs 3-6. The reference compounds for the different sulfur species are shown in parentheses. Error, $\pm 10 \% ;-$, contribution $<0 \cdot 1 \%$.

\begin{tabular}{|c|c|c|c|c|c|c|c|}
\hline \multirow[t]{2}{*}{ Bacterium } & \multicolumn{7}{|c|}{ Percentage contribution of: } \\
\hline & $\begin{array}{l}\mathrm{C}-\mathrm{S}-\mathrm{H} \\
\left(\mathrm{GSH} \mathrm{H}^{*}\right)\end{array}$ & $\begin{array}{l}\mathrm{C}-\mathrm{S}-\mathrm{S}-\mathrm{C} \\
\left(\mathrm{GSSG}{ }^{*}\right)\end{array}$ & $\begin{array}{c}\qquad \mathrm{S}_{2} \mathrm{O}_{3}^{2-} \\
\text { (sodium } \\
\text { thiosulfate) }\end{array}$ & $\begin{array}{c}\mathrm{S}_{4} \mathrm{O}_{4}^{2-} \\
\text { (tetrathionate) }\end{array}$ & $\begin{array}{c}\mathrm{S}_{8} \\
\text { (cyclooctasulfur) }\end{array}$ & $\begin{array}{c}\mathrm{SO}_{4}^{2-} \\
\text { (zinc } \\
\text { sulfate) }\end{array}$ & $\begin{array}{c}\mathrm{S}_{\mu} \\
\text { (polymeric } \\
\text { sulfur) }\end{array}$ \\
\hline Halorhodospira abdelmalekii & 18 & 10 & - & - & - & 5 & 63 \\
\hline Halorhodospira halophila & 12 & 8 & - & - & - & - & 80 \\
\hline Marichromatium purpuratum & - & 18 & - & - & - & 5 & 76 \\
\hline Chlorobium vibrioforme & 3 & 12 & - & - & - & - & 80 \\
\hline Allochromatium vinosum & 15 & 5 & - & - & - & - & 78 \\
\hline Thiocapsa roseopersicina & 37 & 11 & - & - & - & 7 & 44 \\
\hline Thiomargarita namibiensis & - & - & - & - & 95 & - & 5 \\
\hline T. namibiensis (salt water) & - & - & - & - & 77 & 9 & 12 \\
\hline Beggiatoa alba & - & - & - & - & 67 & 21 & 11 \\
\hline Acidithiobacillus ferrooxidans & 10 & - & 8 & 23 & - & - & 58 \\
\hline $\begin{array}{l}\text { Isolated sulfur globules of } A \text {. } \\
\text { vinosum }\end{array}$ & - & - & - & - & 92 & 7 & - \\
\hline
\end{tabular}

* GSH, reduced glutathione; GSSG, oxidized glutathione.

al., 1999). They both store sulfur in globules inside the cells. B. alba stores sulfur in special protein envelopes (Strohl et al., 1981, 1982) while the sulfur is present in the thin outer layer of the cytoplasm in T. namibiensis, which oxidizes sulfide to sulfur while reducing nitrate (Schulz et al., 1999). For Beggiatoa species it is clear that the sulfur is located not in the cytoplasm but in the periplasm of these Gram-negative organisms, while the exact subcellular localization in T. namibiensis has not been determined (Larkin \& Strohl, 1983; Schulz et al., 1999). The sulfur in the globules of both T. namibiensis and $B$. alba appears to consist mainly of cyclooctasulfur. A small minority of longer sulfur chains is also present. This can be deduced from the fact that MINUIT is able to fit the white lines of spectra quite well with only two reference compounds: cyclooctasulfur and polymeric sulfur (Table 3). Sulfate peaks in the spectra $(2481.4 \mathrm{eV})$ and sulfate as indicated by the MINUIT result from salt water in the case of T. namibiensis (Fig. 3, B). In the case of the $B$. alba strain used (which is not able to oxidize sulfur to sulfate) sulfate results from the medium (Fig. 3, C). This medium consists of a saturated calcium sulfate solution (Beggiatoa glides 'fastened on to' the salt crystals) and it was not possible to remove sulfate completely. Our findings support the assumption that these organisms oxidize sulfide to elemental sulfur (cyclooctasulfur) stored in sulfur globules. This could be part of a detoxification strategy in the case of Beggiatoa to cope with sulfide in coastal sediments, their natural environment. Thus, one can assume that the sulfur in 


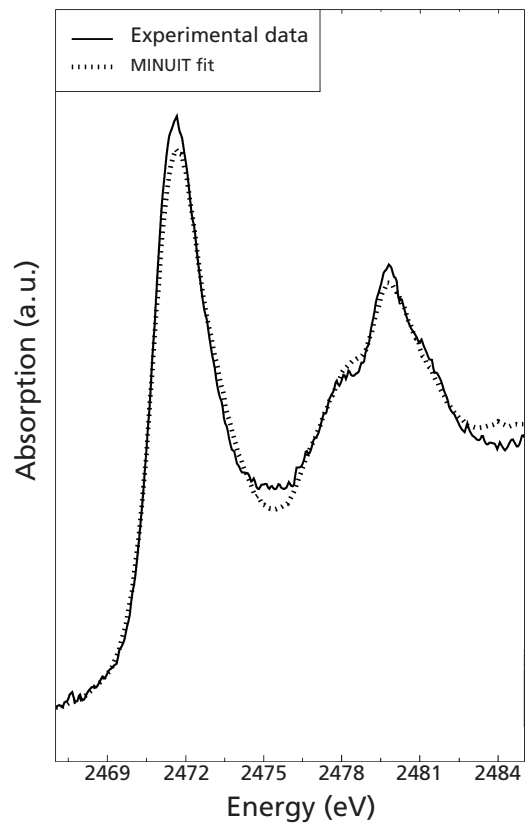

Fig. 4. $\mathrm{S}$ K-edge XANES spectrum of Acidithiobacillus ferrooxidans grown on thiosulfate, and accompanying MINUIT fit. a.u., arbitrary units.

other species of these or closely related genera is also deposited as cyclooctasulfur. Our results and assumptions are supported by a recent Raman spectroscopic analysis of environmental samples of Beggiatoa spp. Raman spectroscopy indicated the same speciation of sulfur ( $\mathrm{S}_{8}$ rings) in Beggiatoa spp. and also in environmental Thioploca spp. samples and in sulfurdepositing endosymbionts of a vesicomyid clam (Pasteris et al., 2001).

\section{Sulfur as polythionates}

The chemotrophic sulfur oxidizer Acidithiobacillus ferrooxidans is able to grow at extremely low $\mathrm{pH}$ values, to oxidize $\mathrm{Fe}^{2+}$ ions or to grow on reduced sulfur compounds (Kelly \& Wood, 2000). When there is a lot of substrate, very small extracellular sulfur globules may be formed and later oxidized to sulfate (Steudel et al., 1987). In Fig. 4, the S K-edge XANES spectrum of $A$. ferrooxidans and accompanying MINUIT fit is shown. The increased noise level of the spectrum of A. ferrooxidans is due to the weak absorption of this sample, which in turn results from the low concentration of small sulfur globules in the culture investigated. We found that sulfur in the sulfur globules of A. ferrooxidans appears as sulfur chains which are most probably terminated by $\mathrm{SO}_{3}^{-}$groups (polythionates), by fitting the whole spectrum on the basis of polymeric sulfur $(\approx 53 \%)$, tetrathionate $(\approx 23 \%)$, reduced glutathione $(\approx 10 \%)$ and thiosulfate $(\approx 8 \%)$ (Table 3$)$. The latter might be a leftover from thiosulfate in the medium. The sulfonate group of the tetrathionate is indicated by

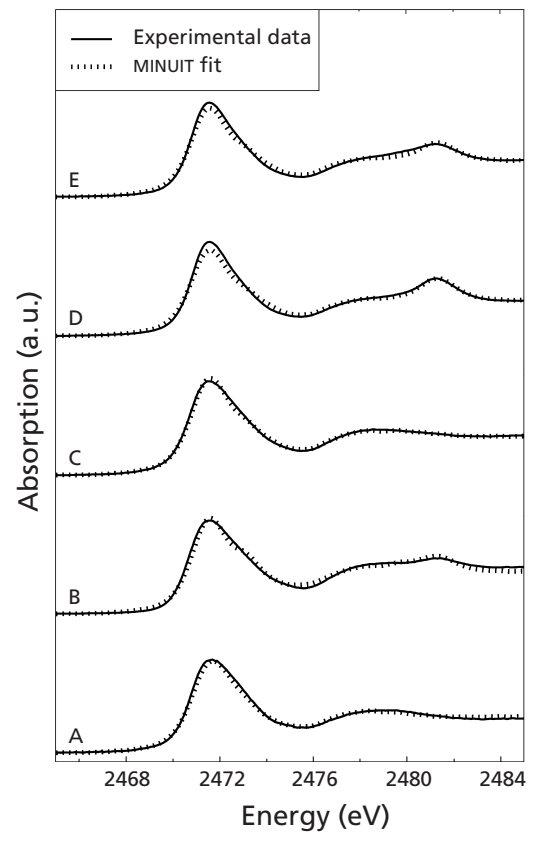

Fig. 5. $\mathrm{S}$ K-edge XANES spectra (normalized on the maximum of the white line) of Halorhodospira halophila (A), Halorhodospira abdelmalekii (B), Chlorobium vibrioforme (C), Thiocapsa roseopersicina (D) and Marichromatium purpuratum (E), and accompanying MINUIT fits. a.u., arbitrary units.

the clear signal at $2480 \cdot 1 \mathrm{eV}$ (Fig. 1b), the typical energy position for sulfonate sulfur. These results support the assumption that polythionates are the dominant sulfur species in sulfur globules of $A$. ferrooxidans. Our finding is in good accordance with previous conclusions based on HPLC analyses of Steudel et al. (1987), who found polythionates with long sulfur chains $\left(\mathrm{S}_{n} \mathrm{O}_{6}^{2-}, n=\right.$ 3 ...22) in cultures of A. ferrooxidans. However, the 'simple sulfur globule model' proposed by Steudel et al. (1987) suggests the presence of polythionates and mainly cyclooctasulfur. MINUIT fitting of the spectrum did not indicate $S_{8}$ rings at all (Table 3), so this model has to be at least partly revised. From our data, we conclude that the sulfur in the globules of A. ferrooxidans mainly consists of polythionates. No significant contribution of cyclooctasulfur (which can be formed in the medium by chemical processes) was found, but its presence cannot be ruled out completely.

\section{Sulfur as sulfur chains}

In Fig. 5, the spectra of different phototrophic sulfur oxidizing bacteria and their accompanying MINUIT fits are shown. All spectra were recorded $1 \mathrm{~h}$ after addition of sulfide. Halorhodospira halophila (A), Halorhodospira abdelmalekii (B) (both Ectothiorhodospiraceae) and Chlorobium vibrioforme (C) (Chlorobiaceae) deposit sulfur outside the cells. The form and position of the white line of the spectra of these bacteria are very similar to those of Thiocapsa roseopersicina (D), Marichromatium purpuratum (E) and Allochromatium vino- 


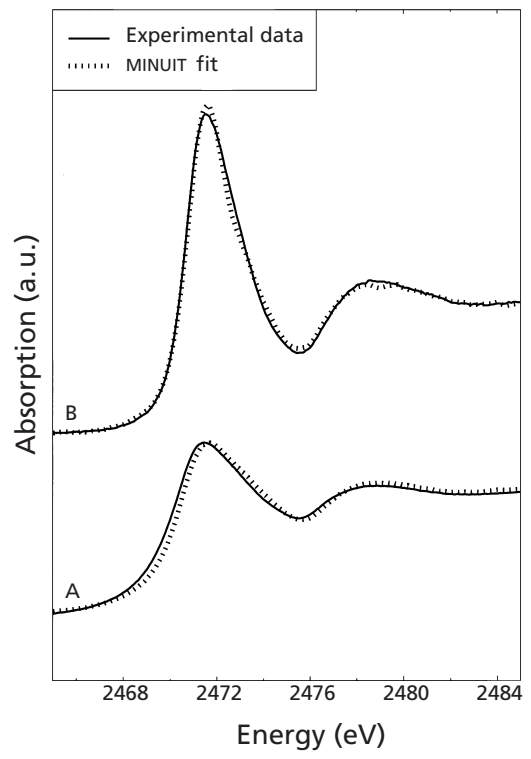

Fig. 6. S K-edge XANES spectra of isolated sulfur globules from Allochromatium vinosum grown on sulfide (A) and intact cells of $A$. vinosum (B), and accompanying MINUIT fits. a.u., arbitrary units.

sum (Fig. 6, B), which belong to the Chromatiaceae and store sulfur inside the cells surrounded by a protein envelope (Brune, 1995; Pattaragulwanit et al., 1998). It may be interesting to note that as in Beggiatoa species the sulfur in A. vinosum and probably also in other members of the Chromatiaceae is not deposited within the cytoplasm but extracytoplasmically inside the chromatophore vesicles that fill up the cells. The interior of these vesicles is topologically equivalent to the periplasm (Pattaragulwanit et al., 1998). The sulfur in all these bacteria mainly consists of sulfur chains $(\approx 80 \%)$ and a minority of (a) substance(s) with $\mathrm{C}-\mathrm{S}-\mathrm{H} /$ C-S-S-C sulfur speciation $(\approx 20 \%$ ) (Table 3$)$. From these results we conclude that either sulfur exists as sulfur chains and an additional 'organic compound' is present, or the sulfur in the sulfur globules is present as mono-/bis-organyl sulfanes in the sulfur globules. The latter possibility might be supported by the fact that fitting of the spectra is also possible using a dioctenyltetrasulfane $\left(\mathrm{R}-\mathrm{S}_{4}-\mathrm{R} ; \mathrm{R}=-\mathrm{C}_{2} \mathrm{H}_{2} \mathrm{C}_{5} \mathrm{H}_{10} \mathrm{CH}_{3}\right)$; however, it achieves only a rather bad agreement (less intense white line) with the experimental data (fits not shown). The effect that increasing the chain length leads to a more intense white line in a $S$ K-edge XANES spectrum is well known from the literature (Sze et al., 1988). Thus the fact that glutathione and polymeric sulfur, the combination of which may represent a long-chained organylsulfane, yield a better fitting result than the tetrasulfane reference might indicate the presence of long sulfur chains $(>3)$ terminated by an appropriate organic residue or hydrogen. This is in good accordance with our previous results and proposals using the 'classical fingerprint method' to interpret bacterial XANES spectra (Prange et al., 1999). However, without the exact reference compound it is not possible to prove this interpretation.

The 'organic compound(s)' and/or 'organic residues' present in the sulfur globules could be responsible for keeping the sulfur in a 'liquid' state at ambient pressure and temperature conditions. That the sulfur is present in a liquid and not a crystalline state has been shown by Hageage et al. (1970) using X-ray diffraction (under wet conditions). Our assumption that organic residues/ compounds are present in phototrophic bacterial sulfur globules is also supported by discrepancies between the density of Allochromatium sulfur globules $\left(1.31 \mathrm{~g} \mathrm{~cm}^{-3}\right.$; Guerrero et al., 1984), which is far lower than that of pure liquid elemental sulfur $\left(1.89 \mathrm{~g} \mathrm{~cm}^{-3}\right.$; Steudel et al., 1990), leading to the proposal of Steudel et al. (1990) that sulfur is complexed with another, less dense component. Interestingly, in a set of reference compounds containing both cysteine/cystine and reduced/ oxidized glutathione, the first pair of references is completely rejected, although both have a comparable atomic environment of the sulfur atom. Furthermore, fitting the spectra with a set of references including cysteine/cystine instead of reduced/oxidized glutathione yields considerably worse agreements of the white lines (data not shown). The need to include glutathione in the basis set can be related to a previous study by Bartsch et al. (1996), who showed the presence of glutathione modified with an amidated glycine carboxylic group and its perthiol in A. vinosum. This led to the speculation that the thiols and perthiols could act as carrier molecules of sulfur to and from the globules (Bartsch et al., 1996; Pott \& Dahl, 1998). The amide group of glutathione amide occurs only in the 7th coordination shell of the sulfur atom, so it should not influence the S K-edge XANES spectrum significantly, i.e. glutathione amide should yield a spectrum virtually identical to that of glutathione. In the case of $T$. roseopersicina (Fig. 5, D), only $\approx 44 \%$ of the sulfur was present as sulfur chains, probably because degradation of the chains had already started. Interestingly, the sulfur in the globules of the extremely halophilic bacterium $H$. abdelmalekii has the same speciation as the other phototrophic bacteria investigated. H. abdelmalekii (growing at $\mathrm{pH}$ 9) oxidizes sulfide to sulfur but is not able to further oxidize elemental sulfur to sulfate (Then \& Trüper, 1983, 1984) so that one could have assumed a different sulfur speciation. Our results, however, indicate a comparable dominating sulfur chain species in the globules of all anoxygenic phototrophic sulfur-accumulating bacteria independent of physiological differences between the bacteria.

\section{Analysis of isolated sulfur globules from Allochromatium vinosum}

Measurements of isolated sulfur globules from anaerobically grown Allochromatium vinosum (globules were isolated $2.5 \mathrm{~h}$ after feeding with sulfide) showed completely different spectra (Fig. 6, A) as compared to those of intact cells (Fig. 6, B). Sulfur was found mainly as cyclooctasulfur $(\approx 92 \%)$ and a minority as sulfate 
$(\approx 7 \%)$, which is found in contrast to the chain sulfur structure for intact cells of A. vinosum (Table 3). During extraction of the globules from the cells, the integrity of the cells was destroyed and oxygen was present (Schmidt et al., 1971), which might have led to spontaneous sulfur ring formation. This shows clearly the necessity of in situ measurements and a non-destructive analysis of the cells, as is the case when XANES spectroscopy is used.

\section{Conclusions}

The spectra and the corresponding quantitative analyses presented in this paper showed clear differences in the speciation of sulfur in the sulfur globules of different metabolic groups of sulfur-oxidizing bacteria, reflecting their ecological and physiological properties. In situ XANES measurements revealed at least three different sulfur species: sulfur rings (cyclooctasulfur) in the microaerobic chemotrophic sulfur-oxidizing bacteria $B$. alba and T. namibiensis, polythionates in the aerobic chemotroph A. ferrooxidans, and sulfur chains in the anaerobically grown phototrophic sulfur bacteria.

Many purple bacteria of the family Chromatiaceae are able to grow chemolithoautotrophically under micro- to semioxic conditions using reduced sulfur compounds as electron donors and oxygen as electron acceptor (Pfennig \& Trüper, 1992). In the future, it will be important to find out whether the sulfur globules formed under these conditions resemble those formed by phototrophically grown cells (sulfur chains) or those formed by chemolithotrophic sulfur bacteria. Such studies would also help to clarify which factors (environmental conditions, metabolic pathways, phylogenetic affiliation of the organisms) govern the speciation of the stored sulfur. Sulfur of isolated sulfur globules from anaerobically grown $A$. vinosum was found as cyclooctasulfur, indicating the necessity of methods like XANES that can be applied to avoid destruction of the original sulfur environment.

\section{ACKNOWLEDGEMENTS}

This study was supported by the Deutsche Forschungsgemeinschaft (grant $\operatorname{Tr} 133 / 26-1,2,3$ ) and the Fonds der Chemischen Industrie (H.G.T.). We would like to thank Heide Schulz for T. namibiensis, Jan Kuever for B. alba and Ralf Steudel for polymeric sulfur. Daniel C. Brune is gratefully acknowledged for useful hints concerning the isolation procedure of sulfur globules. A.P. is indebted to the Stiftung der Deutschen Wirtschaft (Studienförderwerk Klaus Murmann) for a doctoral scholarship and to the Center for Advanced Microstructures \& Devices (CAMD), Louisiana State University, Baton Rouge, for generous financial support of a short-term research visit.

\section{REFERENCES}

Althoff, K. H., von Drachenfels, W., Dreist, A. \& 7 other authors (1990). ELSA - one year experience with the Bonn electron accelerator. Particle Accelerators 27, 347-352.

Bartsch, R. G., Newton, G. L., Sherill, C. \& Fahey, R. C. (1996). Glutathione amide and its perthiol in anaerobic sulfur bacteria. J Bacteriol 178, 4742-4746.
Bianconi, A. (1988). XANES-spectroscopy. In X-ray Absorption: Principles, Applications, Techniques of EXAFS, SEXAFS and XANES, pp. 573-662. Edited by D. C. Koningsberger \& R. C. Prins. New York: Wiley.

Brune, D. C. (1995). Isolation and characterization of sulfur globules proteins from Chromatium vinosum and Thiocapsa roseopersicina. Arch Microbiol 163, 391-399.

Chauvistré, R., Hormes, J., Hartmann, E., Etzenbach, N., Hosch, R. \& Hahn, J. (1997). Sulfur K-shell photoabsorption spectroscopy of the sulfanes R-S $-\mathrm{R}, n=2-4$. Chem Phys 223, 293-302.

Cohn, F. (1875). Untersuchungen über Bakterien. Beitr Biol Pflanz 1, 141-207.

Cramer, C. (1870). In Chemisch-Physikalische Beschreibung der Thermen von Baden in der Schweiz. Edited by C. Müller. Baden: C. Müller.

Donohue, J. (1974). The Structures of the Elements. New York: Wiley.

Guerrero, R., Mas, J. \& Pedrós-Alió, C. (1984). Buoyant density changes due to intracellular content of sulfur in Chromatium warmingii and Chromatium vinosum. Arch Microbiol 137, 350-356.

Hageage, G. J., Jr, Eanes, E. D. \& Gherna, R. L. (1970). X-ray diffraction studies of the sulfur globules accumulated by Chromatium species. J Bacteriol 101, 464-469.

Imhoff, J. F. (1992). The family Ectothiorhodospiraceae. In The Prokaryotes, 2nd edn, pp. 3222-3229. Edited by A. Balows, H. G. Trüper, M. Dworkin, W. Harder \& K. H. Schleifer. New York: Springer.

Imhoff, J. F., Süling, J. \& Petri, R. (1998). Phylogenetic and taxonomic reclassification of Chromatium species and related purple sulfur bacteria. Int J Syst Bacteriol 48, 1129-1143.

Kelly, D. P. \& Wood, A. P. (2000). Reclassification of some species of Thiobacillus to the newly designated genera Acidithiobacillus gen. nov., Halothiobacillus gen. nov. and Thermothiobacillus gen. nov. Int J Syst Evol Microbiol 50, 511-516.

Larkin, J. M. \& Strohl, W. R. (1983). Beggiatoa, Thiothrix and Thioploca. Annu Rev Microbiol 37, 341-367.

Lemonnier, M., Collet, O., Depautex, C., Esteva, J.-M. \& Raoux, D. (1978). High vacuum two crystal soft X-ray monochromator. Nucl Instr Methods 152, 109-111.

Mas, J. \& van Gemerden, H. (1987). Influence of sulfur accumulation and composition of sulfur globule on cell volume and buoyant density of Chromatium vinosum. Arch Microbiol 146, 362-369.

Modrow, H., Visel, F., Zimmer, R. \& Hormes, J. (2001). Monitoring thermal oxidation of sulfur crosslinks in SBR-elastomers by quantitative analysis of sulfur K-edge XANES-spectra. Rubber Chem Technol 74, 281-294.

Parrat, L. G., Hempstead, C. F. \& Jossem, E. L. (1957). 'Thickness effect' in absorption spectra near absorption edges. Phys Rev 105, 1228-1232.

Pasteris, J. D., Freeman, J. J., Goffredi, S. K. \& Buck, K. R. (2001). Raman spectroscopic and laser scanning confocal microscopic analysis of sulfur in living sulfur-precipitating marine bacteria. Chem Geol 180, 3-18.

Pattaragulwanit, K., Brune, D. C., Trüper, H. G. \& Dahl, C. (1998). Molecular genetic evidence for extracytoplasmatic localization of sulfur globules in Chromatium vinosum. Arch Microbiol 169, 434-444.

Pfennig, N. \& Trüper, H. G. (1992). The family Chromatiaceae. In The Prokaryotes, 2nd edn, pp. 3584-3592. Edited by A. Balows, 
H. G. Trüper, M. Dworkin, W. Harder \& K. H. Schleifer. New York: Springer.

Pickering, I. J., Prince, R. C., Divers, T. \& George, G. N. (1998). Sulfur K-edge X-ray absorption spectroscopy for determining the chemical speciation of sulfur in biological systems. FEBS Lett 441, 11-14.

Pickering, I. J., George, G. N., Yu, E. Y., Brune, D. C., Tuschak, C., Overmann, J., Beatty, J. T. \& Prince, R. C. (2001). Analysis of sulfur biochemistry of sulfur bacteria using X-ray absorption spectroscopy. Biochemistry 40, 8138-8145.

Prange, A., Arzberger, I., Engemann, C., Modrow, H., Schumann, O., Trüper, H. G., Steudel, R., Dahl, C. \& Hormes, J. (1999). In situ analysis of sulfur in the sulfur globules of phototrophic sulfur bacteria by X-ray absorption near edge spectroscopy. Biochim Biophys Acta 1428, 446-454.

Prange, A., Kühlsen, N., Birzele, B., Arzberger, I., Hormes, J., Antes, S. \& Köhler, P. (2001). Sulfur in wheat gluten: in situ analysis by X-ray absorption near edge structure (XANES) spectroscopy. Eur Food Res Technol 212, 570-575.

Pott, A. S. \& Dahl, C. (1998). Sirohaem sulfite reductase and other proteins encoded by genes at the $d s r$ locus of Chromatium vinosum are involved in the oxidation of intracellular sulfur. Microbiology 144, 1881-1894.

Rompel, A., Cinco, R. M., Latimer, M. J. \& 7 other authors (1998). Sulfur K-edge X-ray absorption spectroscopy: a spectroscopic tool to examine the redox state of S-containing metabolites in vivo. Proc Natl Acad Sci US A 95, 6122-6127.

Schmidt, G. L., Nicolson, G. L. \& Kamen, M. D. (1971). Composition of the sulfur particle of Chromatium strain D. J Bacteriol 105, 1137-1141.

Schulz, H., Brinkhoff, T., Ferdelman, T. G., Marine, M. H., Teske, A. \& Jørgensen, B. B. (1999). Dense populations of a giant sulfur bacterium in Namibian shelf sediments. Science 284, 493-495.

Siefert, E. \& Pfennig, N. (1987). Convenient method to prepare neutral sulfide solution for cultivation of phototrophic sulfur bacteria. Arch Microbiol 139, 100-101.

Stern, E. A. \& Kim, K. (1981). Thickness effect on the extendedX-ray-absorption-fine-structure amplitude. Phys Rev B 23, 37813787.

Steudel, R. (1989). On the nature of the 'elemental sulfur' $\left(S^{0}\right)$ reduced by sulfur-oxidizing bacteria - a model for $S^{0}$ globules. In Autotrophic Bacteria, pp. 289-303. Edited by H. G. Schlegel \& B. Bowien. Madison, WI: Science Technology Publishers.
Steudel, R. (1996). Das gelbe Element und seine erstaunliche Vielseitigkeit. Chemie in unserer Zeit 30, 226-234.

Steudel, R. \& Albertsen, A. (1999). The chemistry of aqueous sulfur sols - models for bacterial sulfur globules? In Biochemical Principles and Mechanisms of Biosynthesis and Biodegradation of Polymers, pp. 17-26. Edited by A. Steinbüchel. Weinheim: Wiley-VCH.

Steudel, R., Holdt, G., Göbel, T. \& Hazeu, W. (1987). Chromatographic separation of higher polythionates $\mathrm{S}_{n} \mathrm{O}_{6}^{2-}(n=3 \ldots 22)$ and their detection in cultures of Thiobacillus ferrooxidans: molecular composition of bacterial sulfur secretions. Angew Chem Int Ed Engl 26, 151-153.

Steudel, R., Holdt, G., Visscher, P. T. \& van Gemerden, H. (1990). Search for polythionates in cultures of Chromatium vinosum after sulfide incubation. Arch Microbiol 153, 432-437.

Strohl, W. R., Geffers, I. \& Larkin, J. M. (1981). Structure of the sulfur inclusion envelopes from four Beggiatoas. Curr Microbiol 6, 75-79.

Strohl, W. R., Howard, K. S. \& Larkin, J. M. (1982). Ultrastructure of Beggiatoa alba strain B15LD. J Gen Microbiol 128, 73-84.

Sze, K. H., Brion, C. E., Tronc, M., Bodeur, S. \& Hitchcock, A. P. (1988). Inner and valence shell electronic excitation of dimethyl sulfoxide by electron energy loss and photoabsorption spectroscopies. Chem Phys 121, 279-297.

Then, J. \& Trüper, H. G. (1983). Sulfide oxidation in Ectothiorhodospira abdelmalekii. Evidence for the catalytic role of cytochrome c-551. Arch Microbiol 135, 254-258.

Then, J. \& Trüper, H. G. (1984). Utilization of sulfide and elemental sulfur by Ectothiorhodospira halochloris. Arch Microbiol 139, 295-298.

Trüper, H. G. \& Hathaway, J. C. (1967). Orthorhombic sulfur formed by photosynthetic sulphur bacteria. Nature 215, 435-436. Winogradsky, S. N. (1887). Über Schwefelbakterien. Bot Ztg 45, 489-508.

Winter, I., Hormes, J. \& Hiller, M. (1995). Thermal ageing of electrically conducting polymers: XANES measurements of polythiophenes. Nucl Instr Methods B97, 287-291.

Received 18 June 2001; revised 17 September 2001; accepted 26 September 2001. 